\title{
Emergency management of calcium channel blocker overdose
}

\author{
V S Stephen, ${ }^{1,2} \mathrm{MB}$ ChB, MMed (Emergency Medicine), Dip PEC (SA), FCEM (SA); N A Pluymers, ${ }^{1}$ MB ChB, Dip PEC (SA); \\ S J Gauton, ${ }^{3} \mathrm{MB}$ ChB, Dip PEC (SA) \\ ${ }^{1}$ Emergency Department, Thelle Mogoerane Regional Hospital, Vosloorus, South Africa \\ ${ }^{2}$ Division of Emergency Medicine, Faculty of Health Sciences, University of the Witwatersrand, Johannesburg, South Africa \\ ${ }^{3}$ Department of Internal Medicine, Thelle Mogoerane Regional Hospital, Vosloorus, South Africa
}

Corresponding author: VS Stephen (victoria.stephen1@wits.ac.za)

\begin{abstract}
Calcium channel blockers (CCBs) are commonly used in South Africa (SA) in the management of hypertension and other cardiovascular disease. Their ubiquitous availability makes them a common agent in drug overdose (OD), whether through accidental ingestion or deliberate self-harm. It is essential that medical practitioners know how to recognise and manage CCB OD, as severe CCB OD is often fatal. As there is a lack of local literature in SA, we highlight the general principles of management of CCB OD, as well as complications and problems that may be encountered during treatment. This narrative review is based on existing clinical guidelines, retrospective studies and systematic reviews on the emergency management of CCB OD. High-dose insulin euglycaemic therapy has become the mainstay of treatment in severe CCB OD. The rationale, the recommended protocol for its use and its adverse effects are described.
\end{abstract}

S Afr Med J 2019;109(9):635-638. https://doi.org/10.7196/SAMJ.2019.v109i9.13704

Hypertension is a common disease in Africa. A recent study found a prevalence of over $40 \%$ throughout the continent. ${ }^{[1]}$ In South Africa (SA), the current prevalence is estimated to be $30.4 \% \cdot{ }^{[2]}$ Calcium channel blockers (CCBs) have a prominent role in the management of hypertension in SA, and are recommended as first-line agents in black Africans, the elderly, pregnant women, and patients with comorbid diseases such as angina pectoris, peripheral vascular disease and carotid atherosclerosis. ${ }^{[2-6]}$

Drug overdose (OD), whether due to deliberate self-harm or accidental ingestion, is a common patient presentation encountered in the emergency department (ED). ${ }^{[7,8]}$ Medicine-related poisonings predominate and are often severe, requiring emergency care. ${ }^{[7-10]}$ Medications typically reported are those that are easily accessible or are prescribed by healthcare professionals, with prescription drugs identified as being of particular concern in deliberate self-harm. ${ }^{[7]}$ Many of these prescription drugs form part of the essential drugs list, such as CCBs.

Owing to the widespread use of CCBs and a high likelihood of their being available in SA homes, the risk of OD exists. CCBs are highly lethal in severe OD if it is not treated promptly and aggressively. ${ }^{[1]}$ Indeed, CCB ODs are responsible for more fatalities than any other cardiovascular agents in the USA, with an estimated 35\% mortality rate. ${ }^{[12]}$ As there is a lack of local literature in SA, we highlight the general principles of management, complications and problems that may be encountered during treatment. Recommendations are based on available international evidence and current best practice to assist in the emergency management of such cases.

\section{Pharmacology}

CCBs are divided into two main classes, the dihydropyridines (e.g. amlodipine and nifedipine) and the non-dihydropyridines (e.g. verapamil and diltiazem). ${ }^{[11]}$ CCBs directly antagonise the voltagegated L-type calcium channels, which are found in peripheral vascular smooth muscle, cardiomyocytes and the pacemaker cells that form the cardiac conducting system. ${ }^{[11,13,14]}$ Outside the cardiovascular system, voltage-gated L-type calcium channels are found in the beta cells of the pancreas, central nervous system and skeletal muscle. ${ }^{[11,13,14]}$

Non-dihydropyridine CCBs are used primarily to treat angina or cardiac arrhythmias. ${ }^{[11,13]}$ They are lipophilic and have a higher affinity for cardiomyocytes than the dihydropyridine agents, where they reduce the influx of calcium during phase two of the action potential. ${ }^{[13-16]}$ Because calcium is not able to enter the cell, excitation coupling in these cells cannot occur, resulting in reduced myocardial contractility. ${ }^{[15,16]}$ Reduced binding of calcium to the calcium channels in the conducting system leads to slowing of sinoatrial and atrioventricular conduction, resulting in bradycardia. ${ }^{[15,16]}$

The dihydropyridines preferentially affect vascular smooth muscle in the periphery at therapeutic dosages, thereby decreasing peripheral vascular resistance and reducing afterload and lowering blood pressure (BP). ${ }^{[1,13]}$ They are therefore excellent agents for the management of hypertension.

\section{Toxicokinetics}

Amlodipine and other slow-release CCBs are absorbed from the gastrointestinal tract slowly, with peak serum concentrations being reached within $6-12$ hours. ${ }^{[1]}$ In OD, peak concentrations may only be reached at 24 hours. ${ }^{[11,17]}$ It is therefore important to note that patients may appear asymptomatic at the time of presentation, especially when slow-release CCBs are ingested, and then deteriorate 24 hours later ${ }^{[11]} \mathrm{CCBs}$ are primarily metabolised by the cytochrome P450 system. ${ }^{[17]}$ They are highly protein bound and have a high volume of distribution, so are not amenable to removal by haemodialysis. ${ }^{[15,17]}$

\section{Toxicodynamics and pathophysiology}

In OD, non-dihydropyridines cause sinus bradycardia, atrioventricular blocks, hypotension and cardiogenic shock. ${ }^{[11,15,17-19]}$ In dihydropyridine $\mathrm{OD}$, profound peripheral vasodilation produces 
vasoplegic (distributive) shock. ${ }^{[1,15,17-20]}$ A reflex tachycardia fails to occur at toxic doses, as dihydropyridines lose their preferential selectivity for peripheral smooth muscle and instead cause bradycardia and cardiogenic shock. ${ }^{[11,17-20]}$ Dysrhythmias such as atrioventricular blocks may also therefore be seen in dihydropyridine OD. ${ }^{[17,20]}$ The hallmark of CCB OD is therefore circulatory shock due to bradycardia, poor cardiac contractility and profound peripheral vasodilatation.

In addition, CCBs in OD appear to exert toxic effects on L-type calcium channels in the beta cells of the pancreas, preventing insulin release. This results in hypoinsulinaemia and insulin resistance, causing hyperglycaemia and ketoacidosis. ${ }^{[11,15,17,18]}$ In the unstressed state, cardiomyocytes utilise free fatty acids as the primary energy substrate. ${ }^{[17,18]}$ In the stressed state, cardiomyocytes switch to glucose. It is theorised that the hypoinsulinaemic state induced by CCBs prevents glucose uptake into the cardiomyocytes, starving them of their energy substrate, further impairing cardiac contractility. ${ }^{[17,18]}$

The systemic effects of CCB-induced cardiogenic and distributive shock are myriad. Hypoperfusion of the brain from hypotension may cause impairment in consciousness. Occasionally, seizures may occur. ${ }^{[1,17,19]}$ Lactic acidosis develops from the poor perfusion to end organs, as well as reduced conversion of lactate to pyruvate in mitochondria due to reduced entry of calcium into cells. ${ }^{[11,17,18]}$ Acute kidney injury occurs as a result of prolonged hypoperfusion and endorgan ischaemia. ${ }^{[15]}$ Non-cardiogenic pulmonary oedema may occur secondary to pulmonary vasodilation and alveolar extravasation of fluid. ${ }^{[1,15,20]}$ In addition, excessive fluid resuscitation may also contribute to its development. ${ }^{[1,13,17,20]}$

\section{Management of a CCB OD \\ General management, resuscitation and decontamination}

Advice from a poisons information centre should be sought if medical staff are unfamiliar with the management of CCB OD. ${ }^{[11,14,18,20]}$ The Western Cape Poison Information Centre helpline is available 24 hours a day. ${ }^{[21]}$ General resuscitation measures should be started without delay, which include supplemental oxygen, establishment of reliable intravenous access and basic haemodynamic monitoring in the form of heart rate, BP and oxygen saturation. ${ }^{[1,22]}$ This should be done in a closely monitored area, such as the resuscitation area of the ED. ${ }^{[1,18,22]}$ Endotracheal intubation and ventilation may be necessary in severe poisonings where coma and cardiorespiratory failure occur. ${ }^{[11,13]}$ Non-cardiogenic pulmonary oedema can occur in severe poisonings, requiring ventilatory support. ${ }^{[11,13,17]}$ Close monitoring should be continued in the ED until the patient has been transferred to an intensive care unit (ICU) or high-care setting, as a patient may deteriorate precipitously. ${ }^{[1,17,22]}$

If a potentially life-threatening $\mathrm{OD}$ of a sustained-release $\mathrm{CCB}$ has occurred and the patient has presented within 2 hours of ingestion, activated charcoal $1 \mathrm{~g} / \mathrm{kg}$ may be given orally to reduce systemic absorption of the CCB. ${ }^{[14,17,22]}$ Activated charcoal should only be given in fully awake patients who can protect their airway or via a nasogastric tube after the patient has been intubated with an endotracheal tube. ${ }^{[1,17,17,22]}$

The time of ingestion and the number of tablets ingested should be sought. A potentially life-threatening OD varies depending on the type of ССВ ingested. The exact toxic dose for each has not been fully established. However, it is recommended that patients are monitored for toxicity if $>0.3 \mathrm{mg} / \mathrm{kg}$ of amlodipine is ingested in children, ${ }^{[23]}$ and if $>10 \mathrm{mg}$ is ingested by an adult. Asymptomatic patients should be observed for 24 hours in a monitored setting if a sustained-release preparation has been ingested. ${ }^{[1,14,22]}$ Further history taking should include whether concomitant ingestion with other medications, poisons, alcohol or illicit drugs has occurred. Betablocker co-ingestion is common and requires similar management to CCB OD, as described below. ${ }^{[24]}$

CCB toxicity may already be evident on examination on initial presentation. BP may be normal or low with bradycardia. ${ }^{[11,17]}$ Signs of distributive shock may be present, such as bounding pulses and warm peripheries, particularly in dihydropyridine OD. ${ }^{[11,14,17,19]}$ These clinical signs should not be mistaken for adequate perfusion. The patient may have bounding pulses from peripheral vasodilation, so the severity of shock may be underestimated and unrecognised until decompensation occurs, whereupon the patient's haemodynamics may deteriorate rapidly. ${ }^{[18]}$ Pulmonary oedema may already be present on initial presentation if presentation to hospital was delayed. ${ }^{[13]}$

Bedside investigations include a 12-lead electrocardiogram (ECG) to look for arrhythmias. ${ }^{[1,17,22]}$ Venous blood gas analysis is helpful to confirm the presence of metabolic acidosis and lactic acidosis, ${ }^{[1,17,18]}$ as well as to check the baseline serum potassium. ${ }^{[25]}$ Females of reproductive age should have a urine pregnancy test. ${ }^{[26]}$ Pregnant patients may require specific treatment, such as being nursed in a leftlateral position to reduce the effects of supine hypotension syndrome caused by compression of the inferior vena cava by the uterus, which may further exacerbate the shock induced by CCB OD. ${ }^{[26]}$

A serum paracetamol measurement should be done if concomitant medications ingested are not known, as paracetamol is the most common medication used in OD in SA, lacks a clinically detectable toxidrome, and requires specific life-saving management. ${ }^{[27]}$ Other serum drug levels should be guided by clinical suspicion. ${ }^{[28]}$

\section{Specific management in the symptomatic CCB OD}

Two intravenous lines should be established promptly, and $20 \mathrm{~mL} /$ $\mathrm{kg}$ crystalloid can be given initially if shock is present. ${ }^{[14,15,17,19,22,24]}$ Avoid further excessive intravenous fluid administration, as this is associated with an increased risk of pulmonary oedema. ${ }^{[11,15,20]}$ Central venous access is extremely useful, as all but mild poisonings will require multiple infusions to run simultaneously, and frequent blood gas monitoring is required. ${ }^{[25]}$ Access should be obtained as soon as feasible.

Thirty to sixty millilitres of $10 \%$ calcium gluconate or $10-20 \mathrm{~mL}$ $10 \%$ calcium chloride may be given intravenously over 10 - 20 minutes, although clinical evidence of improvement is limited. ${ }^{[11,14,15,17-19,22,24]}$ Atropine in 0.5 - $1 \mathrm{mg}$ boluses intravenously, up to a maximum of $3 \mathrm{mg}$, may be tried for CCB-induced bradycardia. ${ }^{[14]}$ Glucagon has no proven role in CCB OD, although it may be of benefit in beta-blocker ODs if sufficient quantities can be sourced. ${ }^{[11,14,18,24]}$

Patients with signs of shock such as mean arterial pressure (MAP) $<65 \mathrm{mmHg}$ or lactic acidosis despite initial fluid resuscitation should be started on an adrenergic agent. ${ }^{[12,14,15,17-19,22,24]}$ In the SA setting, adrenaline is most commonly available. Adrenaline has positive inotropic and chronotropic effects as well as vasopressor effects in higher doses. ${ }^{[14,17]}$ The dosage range for infusion is between 0.05 and $1 \mu \mathrm{g} / \mathrm{kg} / \mathrm{min}$, aiming for improvement in haemodynamics and perfusion. ${ }^{[14]}$ A good starting point for a hypotensive patient with CCB OD is $0.5 \mu \mathrm{g} / \mathrm{kg} / \mathrm{min}$, which can be up- or down-titrated to effect. Dopamine is generally not recommended. ${ }^{[22]}$ In severely shocked patients with CCB poisoning, adrenergic agents such as adrenaline may not reverse shock adequately. ${ }^{[14,17,19,29-31]}$

\section{High-dose insulin euglycaemic therapy}

If the patient is not demonstrating haemodynamic improvement with adrenaline, high-dose insulin euglycaemic therapy (HIET) should be initiated while the vasopressor infusion is continued. ${ }^{[13,14,17,22]}$ There is 
no evidence to recommend a specific timeframe, although initiating HIET 15 minutes after the vasopressor infusion has shown no improvement may be reasonable. Delayed HIET has been associated with increased mortality. ${ }^{[17,18,24,30]}$

There are no randomised controlled studies in humans comparing adrenergic agents with HIET, but animal studies and case studies in humans consistently show HIET to be superior to adrenergic agents in terms of haemodynamic effects in severe CCB OD and shock. ${ }^{[14,17,18,24,25,29-32]}$ HIET has been shown to restore haemodynamic stability where high doses of multiple vasopressors have failed. ${ }^{[1,14,15,18,19,29,30]}$ In multiple case studies it has been demonstrated that when HIET was initiated in CCB-induced shock, it was possible to wean patients off the high-dose vasopressors they had previously required. ${ }^{[13-15,18,19,31]}$ For this reason, HIET is increasingly being advocated for consideration as first-line therapy, ${ }^{[18,19,25,30,31]}$ although further evidence is required to make this standard practice.

The effect of HIET is usually seen 1 hour after initiation, ${ }^{[11,14,17,18,24]}$ so adrenaline should be continued until the patient's haemodynamics have normalised. HIET should not be delayed until ICU admission, as delayed HIET has been associated with increased mortality, as mentioned above. ${ }^{[17,18,24,30]}$

The mechanism for the efficacy of HIET, also known as highdose insulin therapy, is not fully understood. Its efficacy may be explained in three ways. Firstly, insulin acts as a catecholamineindependent inotrope directly on cardiomyocytes. ${ }^{[17,25,31]}$ Secondly, insulin enables the uptake of glucose into cardiomyocytes, which is the heart's preferred energy substrate during stress. ${ }^{[17,18,25,30,31]}$ Since CCBs in OD produce a hypoinsulinaemic state, glucose uptake into the cardiomyocytes cannot occur, impairing cardiac contractility. Exogenous high-dose insulin and supplemental glucose therefore enable uptake of glucose and therefore improve myocardial contractility. ${ }^{[17,18]}$ Finally, insulin acts as a peripheral vasodilator reducing afterload, which improves systemic perfusion. ${ }^{[30,31]}$ In the coronary arteries, this vasodilatory effect improves perfusion and oxygen delivery to the heart, improving inotropy. ${ }^{[25,30,31]}$

HIET is commenced with a loading dose of $1 \mathrm{IU} / \mathrm{kg}$ short-acting insulin intravenously ${ }^{[1,14,17,22,24,25,30,31]}$ (see 'Practical tips' box). An infusion of insulin is commenced simultaneously, ranging from $1 \mathrm{IU} /$ $\mathrm{kg} / \mathrm{h}$ to $10 \mathrm{IU} / \mathrm{kg} / \mathrm{h}$, aiming for haemodynamic stability. ${ }^{[17,22,25]}$ The rate of HIET should be increased up to the maximum dose if shock persists. ${ }^{[1,14,17,18,22,24]}$ In case reports, rates of up to $22 \mathrm{IU} / \mathrm{kg} / \mathrm{h}$ have been used safely and effectively. ${ }^{[1,24]}$ Target haemodynamic parameters include MAP $>65 \mathrm{mmHg},{ }^{[30]}$ systolic $\mathrm{BP}>90 \mathrm{mmHg}$ and urine output $1-2 \mathrm{~mL} / \mathrm{kg}$ in adults. ${ }^{[18,25]}$ Higher MAPs and systolic BPs may be considered in patients known to have poorly controlled hypertension.

At the same time as the loading dose of short-acting insulin, a loading dose of $1 \mathrm{~mL} / \mathrm{kg}$ of $50 \%$ dextrose water is given. ${ }^{[1,17,22]}$ A $10 \%$ dextrose infusion should be commenced to maintain normoglycaemia, and fingerprick glucose testing should be done 30 minutes after HIET is started and hourly thereafter. ${ }^{[1,14,17,23]}$ Higher concentrations of dextrose water, for example $50 \%$, may be required. ${ }^{[25]}$ However, hyperglycaemia despite high doses of HIET can occur $^{[24,25]}$ and may indicate the severity of CCB poisoning. ${ }^{[17]}$

Intravenous potassium replacement should also be commenced once the serum potassium level is $<3.5 \mathrm{mmol} / \mathrm{L}$, similar to the management of diabetic ketoacidosis. ${ }^{[1,14,24,25]}$ A recommended infusion rate for potassium can be found in the accompanying HIET protocol (see box).

The rationale for HIET should be thoroughly explained to medical and nursing staff looking after the patient, as the high doses required may be assumed to be a drug error and stopped, leading to worsened haemodynamic instability and death. ${ }^{[18,25,31]}$

Patients require intensive care or high-care admission owing to the intensive monitoring and multiple infusions they may require. ${ }^{[11,18,22]}$ Transfer to an ICU may occur provided that the patient is haemodynamically stable enough to tolerate transport out of the ED. Transfer to another hospital capable of providing ICU facilities may be required if the patient initially presents to a facility lacking these resources.

\section{Complications during treatment}

Hypokalaemia and hypoglycaemia are two expected complications of HIET and must be monitored for and treated diligently. ${ }^{[1,14,17,22-25]}$ Hypokalaemia and hypoglycaemia are not indications to stop HIET if haemodynamic stability has not yet been achieved. Hypokalaemia is common and seldom life threatening provided that potassium replacement is continued during HIET and serial serum potassium monitoring is done. ${ }^{[14,18,24]}$ Venous blood gas monitoring is done at

\section{Practical tips for managing calcium channel blocker overdose}

Western Cape Poison Information Centre 24-hour helpline: 0861-555-777 or (021) 938-4497

\section{Recommended high-dose insulin euglycaemic therapy protocol}

1. Loading dose of $50 \%$ dextrose $1 \mathrm{~mL} / \mathrm{kg}$ intravenously.

2. Loading dose of short-acting insulin $1 \mathrm{IU} / \mathrm{kg}$ intravenously.

3. Mix insulin infusion: Withdraw $20 \mathrm{~mL}$ from $200 \mathrm{~mL} \mathrm{0.9 \%} \mathrm{saline} \mathrm{bag.} \mathrm{Insert} 2000 \mathrm{IU}$ of short-acting insulin into the bag. Concentration is $10 \mathrm{IU} / \mathrm{mL}$. Start infusion at $1 \mathrm{IU} / \mathrm{kg} / \mathrm{h}$.

4. Commence $10 \%$ dextrose water infusion to maintain normoglycaemia. This may be changed to a $50 \%$ dextrose infusion if required. The rate of dextrose is titrated based on hourly fingerstick glucose measurements. Administer $1 \mathrm{~mL} / \mathrm{kg} 50 \%$ dextrose if blood glucose $<3$ $\mathrm{mmol} / \mathrm{L}$

5. Check fingerstick glucose 30 minutes after infusion is commenced. Continue to check hourly thereafter.

6. Do venous blood gas analysis on arrival and then 4 - 6-hourly.

7. Start potassium chloride infusion if serum potassium is $<3.5 \mathrm{mmol} / \mathrm{L}$, aiming for a serum potassium level of $2.8-4.5 \mathrm{mmol} / \mathrm{L}$. Recommended rate $10 \mathrm{mmol} / \mathrm{h}$, maximum rate $20 \mathrm{mmol} / \mathrm{h} \cdot{ }^{[34]}$ Potassium infusions should run through a central line to avoid phlebitis and patient discomfort.

8. Serum magnesium should be checked and replaced if low.

9. Increase insulin infusion up to $10 \mathrm{IU} / \mathrm{kg} / \mathrm{h}$ until haemodynamic stability is achieved, i.e. mean arterial pressure $>60 \mathrm{mmHg}$, heart rate $>60 \mathrm{bpm}$, normal sinus rhythm on electrocardiogram, urine output $1 \mathrm{~mL} / \mathrm{kg} / \mathrm{h}$, lactate $<2 \mathrm{mmol} / \mathrm{L}$. 
least 4 - 6-hourly during the first $24-48$ hours of therapy. ${ }^{[18,25,30]}$ Supplemental potassium infusions should be stopped once the patient has been weaned off the HIET, because the patient is not inherently potassium deficient. ${ }^{[14,18]}$

It is also essential to monitor for hypoglycaemia during the course of treatment. ${ }^{[18,25,30]}$ Supplemental 50\% dextrose water boluses may be given additionally to maintain normoglycaemia. ${ }^{[1,18,24,25]}$ The importance of strict glucose monitoring should be emphasised to medical staff, particularly in intubated patients, to avoid unrecognised neuroglycopenia.

There is limited consensus in the literature as to when HIET can be stopped, although early or abrupt withdrawal of HIET is associated with rebound of CCB toxicity..$^{[18,24,25]}$ HIET should not be stopped until haemodynamic stability is achieved and the patient no longer requires inotropic support with adrenaline. HIET should be weaned slowly, and this may be days after initiation. ${ }^{[24,25]}$ The end-points of HIET therapy include maintenance of MAP $>65 \mathrm{mmHg}$, heart rate $>60 \mathrm{bpm}$, urine output $1-2 \mathrm{~mL} / \mathrm{kg}$, eradication of lactic acidosis, and improvement in the level of consciousness. ${ }^{[18,25,30,33]}$ Psychological support and psychiatric evaluation are indicated in cases of deliberate self-harm once the patient has been fully treated and stabilised.

\section{The patient in refractory shock and the patient in cardiac arrest}

Effective cardiopulmonary resuscitation and attention to advanced cardiac life support principles remain the mainstay of therapy in CCB-induced cardiac arrest. ${ }^{[14,23]}$ In refractory shock, ensure that the maximum doses of HIET, up to $10 \mathrm{IU} / \mathrm{kg} / \mathrm{h}$, have been used; however, even higher doses have been used safely. ${ }^{[18,22,24]}$ Intravenous lipid emulsion therapy (ILE) may be tried in the peri-arrest or arrested patient, although evidence of efficacy is scarce. ${ }^{[14,22,24]}$ ILE binds to lipophilic drugs, reducing their free concentration in target organs. ${ }^{[11]}$ A loading dose of $1.5 \mathrm{~mL} / \mathrm{kg}$ of $20 \%$ lipid emulsion is given, followed by an infusion of $0.25 \mathrm{~mL} / \mathrm{kg} / \mathrm{m}$ over $30-60$ minutes. ${ }^{[14]}$ Lipid emulsion may be found in theatre, where it is used in cases of local anaesthetic-induced cardiotoxicity. Finally, venoarterial extracorporeal membrane oxygenation is a last resort for patients in refractory shock where this resource is available. ${ }^{[1,14,24]}$

\section{Conclusions}

CCBs are commonly used in SA in the management of hypertension and other cardiovascular disease. It is essential that medical practitioners know how to recognise and manage CCB OD, as severe CCB OD is often fatal. The Poison Information Centre in Cape Town has a 24-hour helpline to assist practitioners with toxicology management. Haemodynamic monitoring and general resuscitation measures should be instituted without delay in patients who are symptomatic. Asymptomatic patients should be monitored closely for at least 24 hours, particularly if a slow-release CCB has been ingested. Intravenous fluid, calcium and vasopressors form part of the initial management of CCB-induced shock. HIET should be started in shocked patients who do not rapidly respond to initial therapy. HIET can be safely used in a monitored, high-care setting. Medical staff should be educated about the rationale of the high doses used to avoid premature stopping of therapy and its adverse consequences.

\section{Acknowledgements. None.}

Author contributions. The authors contributed equally to the writing, editing and revising of the manuscript.

Funding. None.

Conflicts of interest. None.
1. Gómez-Olivé F, Ali S, Made F, et al. Regional and sex differences in the prevalence and awareness of hypertension across six sites in sub-Saharan Africa: An H3Africa AWI-Gen study. Reg Glob Heart 2017:12(2):81-90.

2. Seedat Y, Rayner B, Veriava Y. South African Hypertension Practice Guideline 2014. Cardiovasc J Afr 2014;25(6):288-294. https://doi.org/10.5830/CVJA-2014-062

3. Rayner B, Spence J. Hypertension in blacks. J Hypertens 2017;35(2):234-239. https://doi.org/10.1097/ HJH.0000000000001171

4. Rayner B. Hypertension: Detection and management in South Africa. Nephron Clin Pract 2010;116(4):c269-c273. https://doi.org/10.1159/000318788

5. Opie L, Seedat Y. Hypertension in sub-Saharan African populations. Circulation 2005;112(23):35623568. https://doi.org/10.1161/CIRCULATIONAHA.105.539569

6. Brewster L, Seedat K. Why do hypertensive patients of African ancestry respond better to calcium blockers and diuretics than to ACE inhibitors and $\beta$-adrenergic blockers? A systematic review. BMC Med 2013;11:141. https://doi.org/10.1186/1741-7015-11-141

7. Veale D, Wium C, Müller G. Toxicovigilance I: A survey of acute poisoning in South Africa based on Tygerberg Poison Information Centre data. S Afr Med J 2013;103(5):293-297. https://doi.org/10.7196/ Tygerberg Poison.6647

8. Veale D, Wium C, Müller G. Toxicovigilance II: A survey of the spectrum of acute poisoning and current practices in the initial management of poisoning cases admitted to South African hospitals. S Afr Med J practices in the initial management of poisoning cases adn
2013;103(5):298-303. https://doi.org/10.7196/SAMJ.6648

9. Ani J, Ross A, Campbell L. A review of patients presenting to accident and emergency department with deliberate self-harm, KwaZulu-Natal, South Africa. Afr J Prim Health Care Fam Med 2017;9(1):a1234. https://doi.org/10.4102/phcfm.v9i1.1234

10. Bantjes J, Breedt E, de Wet H, Khan M, Weiss R, Lewis I. Gender differences in epidemiology and medical service utilisation among self-harm patients seeking treatment at an urban hospital in South Africa. Suicidol Online 2017;8(1):10-23. http://www.suicidology-online.com/pdf/Issue\%202017\%20Vol\%20 8\%20(1).pdf (accessed 31 July 2019).

11. Rietjens SJ, de Lange DW, Donker DW, Meulenbelt J. Practical recommendations for calcium channel antagonist poisoning. Neth J Med 2016;74(2):60-67. http://www.njmonline.nl/getpdf.php?id=1674 (accessed 7 August 2019).

12. Watson WA, Litowitz TL, Klein-Schwartz W, et al. 2003 annual report of the American Association of Poison Control Centers toxic exposure surveillance system. Am J Emerg Med 2004;22(5):335-404. https://doi.org/10.1016/j.ajem.2004.06.001

13. Siddiqi TA, Hill J, Huckleberry Y, Parthasarathy S. Non-cardiogenic pulmonary edema and life3. Siddiqi TA, Hill J, Huckeberry Y, Parthasarathy S. Non-cardiogenic pulmonary edema and life-
threatening shock due to calcium channel blocker overdose: A case report and clinical review. Respir threatening shock due to calcium channel blocker overdose: A
Care 2014;59(2):el5-e21. https://doi.org/10.4187/respcare.02244

14. Graudins A, Lee H, Druda D. Calcium channel antagonist and beta-blocker overdose: Antidotes and adjunct therapies. Br J Clin Pharmacol 2016;81(3):453-461. https://doi.org/10.1111/bcp.12763

15. Kumar K, Biyyam M, Bajantri B, Nayudu S. Critical management of severe hypotension caused by amlodipine toxicity managed with hyperinsulinemia/euglycemia therapy supplemented with calcium gluconate, intravenous glucagon and other vasopressor support: Review of literature. Cardiol Res 2018;9(1):46-49. https://doi.org/10.14740/cr646w

16. Silverthorn D. Cardiovascular physiology. In: Espinoza D, German K, Yien B, Reid A, Cutt S, Earl W, eds. Human Physiology: An Integrated Approach. 5th ed. San Francisco: Pearson Benjamin Cummings, 2010:467-511.

17. DeRoos F. Calcium channel blockers. In: Hoffman R, ed. Goldfranks Toxicologic Emergencies. 10th ed. New York: McGraw-Hill, 2015:884-892.

18. Nickson CP, Little M. Early use of high-dose insulin euglycaemic therapy for verapamil toxicity. Med J Aust 2009;191(6):350-352. https:///oi.org/10.5694/j.1326-5377.2009.tb02822.x
Aickson CP. Little M. Early use of high-dose insulin euglycaemic therapy for

19. Rizvi I, Ahmad A, Zaman S. Life-threatening calcium channel blocker overdose and its management. 9. Rizvi l, Ahmad A, Zaman S. Life-threatening calcium chann
BMJ Case Rep 2012. https://doi.org/10.1136/bcr.01.2012.5643

20. Miranda C, Xaivier L, Fiorante F, et al. Cardiac rhythm disturbances associated with amlodipine acute . Miranda C, Xaivier L, Fiorante F, et al. Cardiac rhythm disturbances associated with amlodipine
intoxication. Cardiovasc Toxicol 2012;12(4):359-362. https://doi.org/10.1007/s12012-012-9176-9

21. Department of Paediatrics and Child Health, University of Cape Town. Poisons Information Centre. http://www.paediatrics.uct.ac.za/poisons-information-centre (accessed 29 September 2018).

22. St-Onge M, Anseeuw K, Cantrell FL, et al. Experts consensus recommendations for the management of calcium channel blocker poisoning in adults. Crit Care Med 2017;45(3):e306-e315. https://doi. org/10.1097/CCM.0000000000002087

23. Benson BE, Spyker DA, Troutman WA, Watson WA, Bakhireva LN. Amlodipine toxicity in children less than 6 years of age: A dose-response analysis using national poison system data. J Emerg Med 2010;39(2):186-193. https://doi.org/10.1016/j.emermed.2009.02.16

24. Woodward C, Pourmand A, Mazer-Amirshai M. High-dose insulin therapy, an evidence-based approach to beta-blocker/calcium channel blocker toxicity. Daru J Pharm Sci 2014;22(1):36. https://doi. org/10.1186/2008-2231-22-36

25. Cole J, Arens A, Laes J, Klein L, Bangh S, Olives T. High dose insulin for beta-blocker and calcium channel-blocker poisoning. Am J Emerg Med 2018;36(10):1817-1824. https://doi.org/10.1016/j. ajem.2018.02.004

26. Nelson L, Lewin N, Howland M, Hoffman R, Goldfrank L, Flomenbaum N. Principles of managing the acutely poisoned or overdosed patient. In: Hoffman R, ed. Goldfrank's Toxicologic Emergencies. 10th ed. New York: McGraw-Hill, 2015:37-44

27. Van Hoving D, Hunter L, Gerber R, Lategan H, Marks C. The burden of intentional self poisioning on a district-hospital public hospital in Cape Town, South Africa. Afr J Emerg Med 2018;8(3):79-83. https:// doi.org/10.1016/j.afjem.2018.03.002

28. Rainey P. Laboratory principles. In: Hoffman R, ed. Goldfrank's Toxicologic Emergencies. 10th ed. New York: McGraw-Hill, 2015:70-89.

29. Skoog C, Engebretsen K. Are vasopressors useful in toxin-induced cardiogenic shock? Clin Toxicol (Phila) 2017;55(4):285-304. https://doi.org/10.1080/15563650.2017.1284329

30. Engebretsen K, Kaczmarek K, Morgan J, Holger J. High-dose insulin therapy in beta-blocker and calcium channel-blocker poisoning. Clin Toxicol (Phila) 2011;49(4):277-283. https://doi.org/10.3109/155636 50.2011 .582471

31. Seegobin K, Maharaj S, Deosaran A, Reddy P. Severe beta blocker and calcium channel blocker overdose: Role of high dose insulin. Am J Emerg Med 2018;36(4):736.e5-736.e6. https://doi.org/10.1016/j.
Roteger ajem.2018.01.038

32. St-Onge M, Dubé $P$, Gosselin $S$, et al. Treatment of calcium channel blocker poisoning: A systematic review. Clin Toxicol (Phila) 2014;52(9):926-944. https://doi.org/10.3109/15563650.2014.965827

33. Cecconi M, de Backer D, Antonelli M, et al. Consensus on circulatory shock and haemodynamic monitoring. Task force of the European Society of Intensive Care Medicine. Intensive Care Med 2014;40(12):1795-1815. https://doi.org/10.1007/s00134-014-3525-z

34. National Department of Health, South Africa. Essential Drugs Programme. Hospital Level (Adults) Standard Treatment Guidelines and Essential Medicines List. 4th ed. Pretoria: NDoH, 2015. http://www. health.gov.za/index.php/standard-treatment-guidelines-and-essential-medicines-list/category/286hospital-level-adults?download=2409:hospital-level-adult-2015-v5-0 (accessed 23 November 2018). 